
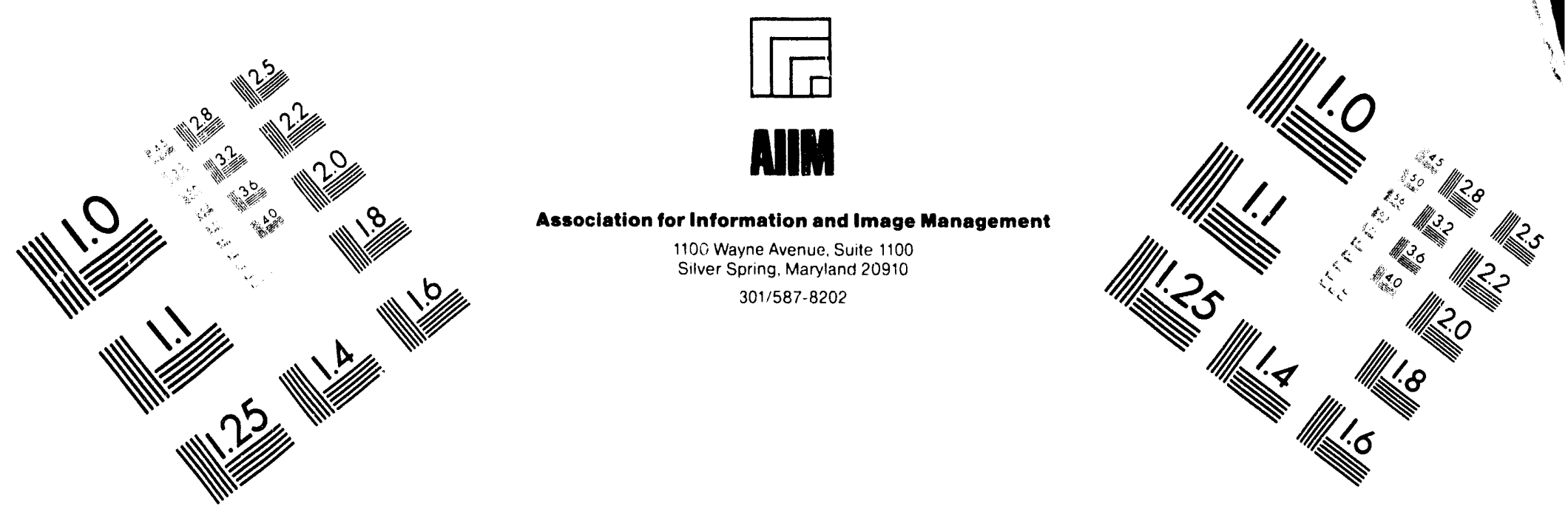

\title{
Centimeter
}

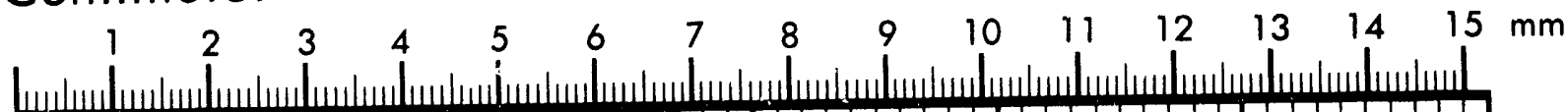

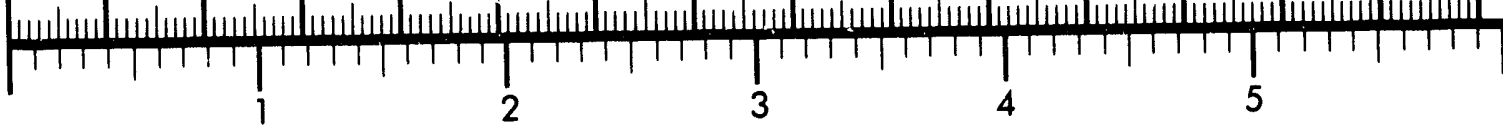
Inches
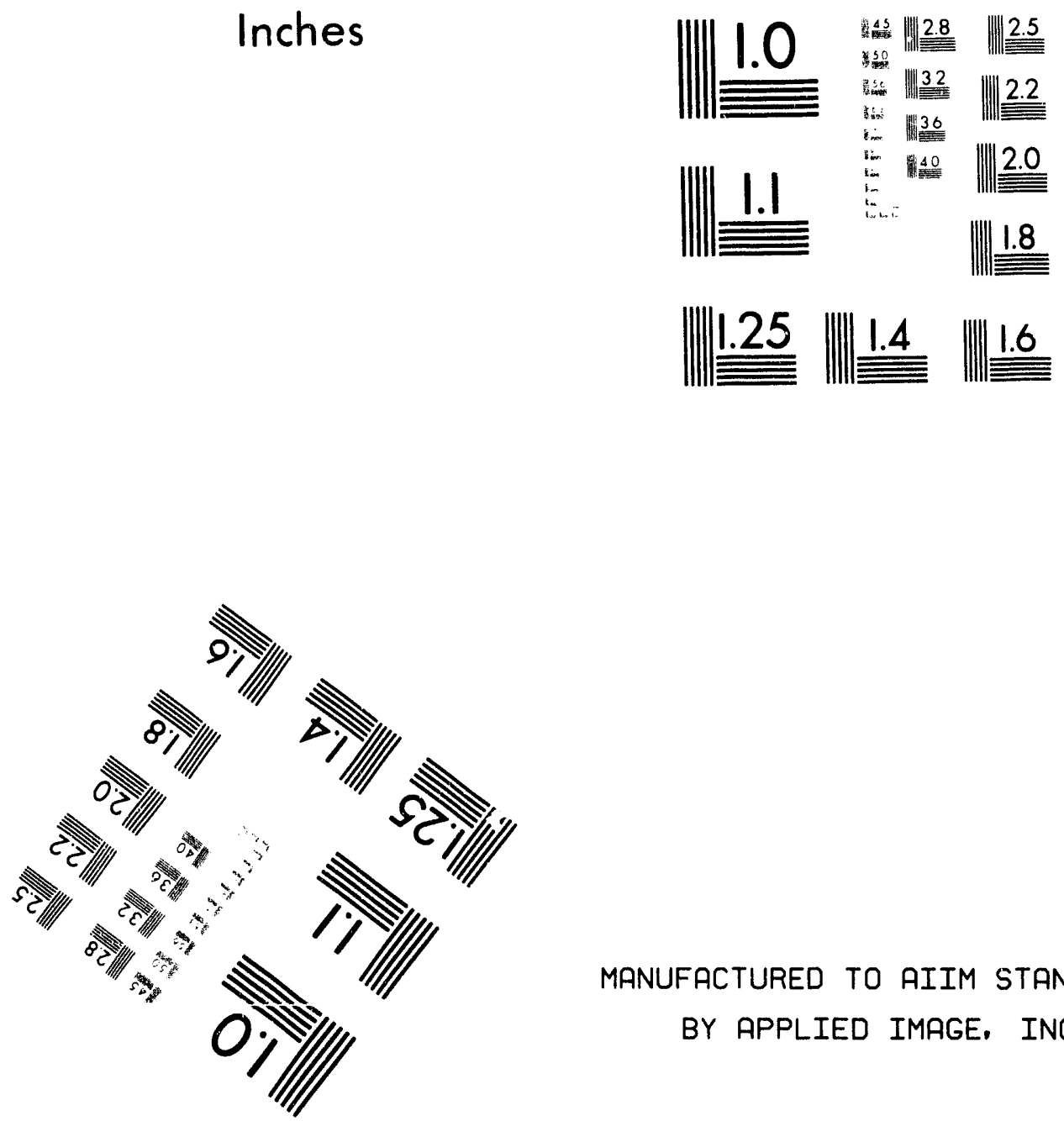

MANUFACTURED TO AIIM STANDARDS

BY APPLIED IMAGE, INC.

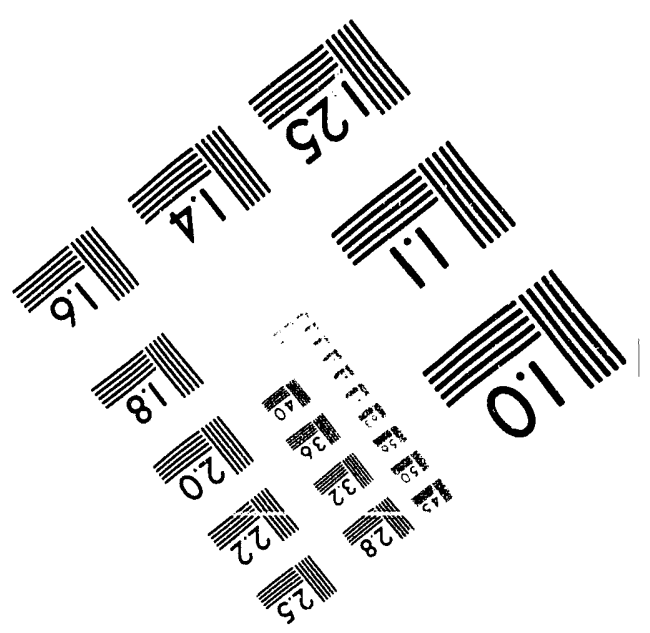



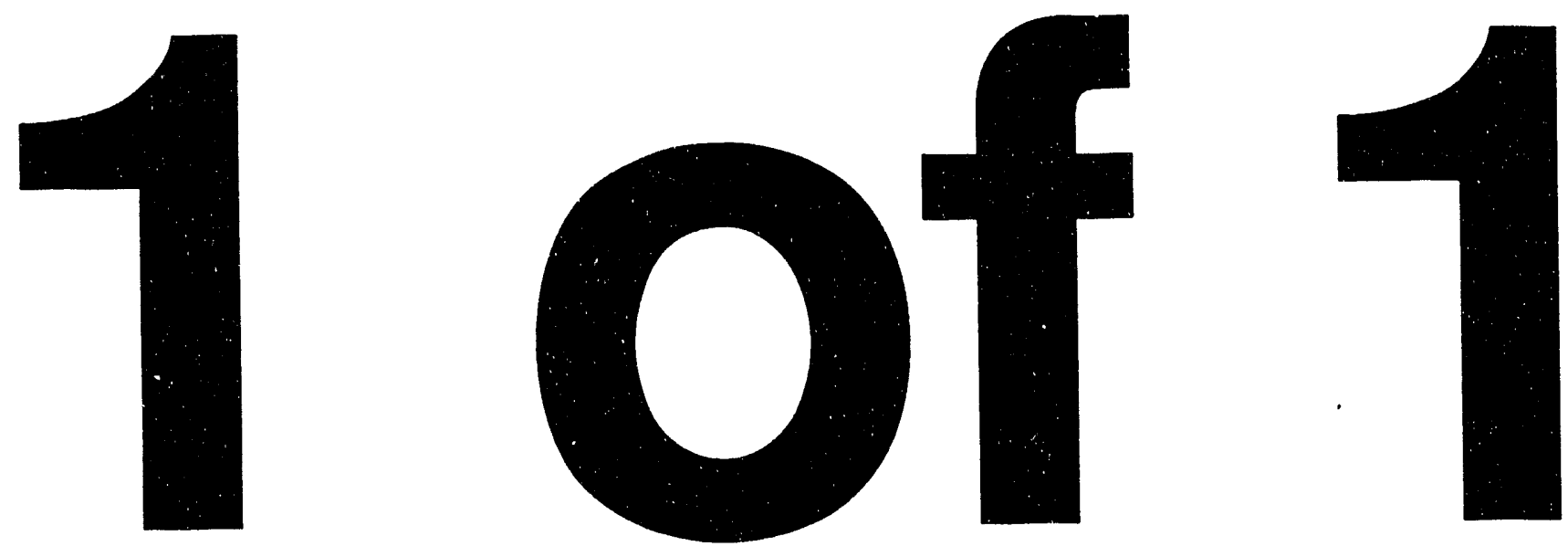
LBL-34143

UC-404

\section{Defects in GaAs Bulk Crystals and Multi-Layers Caused by In Diffusion}

P. Werner, ${ }^{1}$ Z. Liliental-Weber, W. Swider, H. Sohn, ${ }^{+}$ W. Yau, ${ }^{+}$J. Baranowski, ${ }^{2}$ and E.R. Weber ${ }^{+}$

${ }^{1}$ Max Planck Institute of Microstructure Physics Halle/Saale, Germany

Center for Advanced Materials, Materials Sciences Division Lawrence Berkeley Laboratory, University of California Berkeley, CA 94720 USA

+Department of Materials Sciences

University of California

Berkeley, CA 94720 USA

${ }^{2}$ Physics Department, University of Warsaw

Warsaw, Poland

April 1993

This work was supported by the Director, Office of Energy Research, Office of Basic Energy Sciences, Materials Sciences Division, of the U.S. Department of Energy under Contract No. DE-AC03-76SF00098. 


\section{DEFECTS IN GaAs BULK CRYSTALS AND MULTI-LAYERS CAUSED BY In DIFFUSION}

P.Werner, ${ }^{1}$ Z.Liliental-Weber, W. Swider, H.Sohn, ${ }^{+}$WaiFan Yau, J.Baranowski, ${ }^{2}$ and E.R.

Weber, +

Center for Advanced Materials, Materials Science Division, Lawrence Berkeley Laboratory, 62/203, Berkeley, CA 94720, and +Dept.of Materials Science, University of California, Berkeley, CA 94720.

${ }^{1}$ Current address: Max Planck Institute of Microstructure Physics, Halle/Saale, Germany,

2Current address: Physics Department, University of Warsaw, Poland

\section{ABSTRACT}

The objective of this work was to study by transmission electron microscopy the lattice defects in GaAs bulk crystals and heterostructures formed by In diffusion. In such samples hints for the existence of superconductivity have been found. Indium was found to move more than $100 \mu \mathrm{m}$ into bulk GaAs during $1 \mathrm{~h}$ annealing at $550^{\circ} \mathrm{C}$ (such conditions are typical for molecular beam epitaxy growth on GaAs wafers). This rapid diffusion is accompanied by the creation of dislocation networks and metallic In droplets that show evidence for lattice strain. To study the interaction of In with the GaAs lattice, In/GaAs multi-layers were grown by MBE at about $450^{\circ} \mathrm{C}$ on a $\mathrm{GaAs}$ buffer layer. The interfaces of these structures showed misfit dislocations at islands of InAs besides the presence of lattice strain. Both types of samples showed microwave absorption signals typical for superconductivity. The most likely superconductive phases are small metastable inclusions, probably consisting amorphous $\mathrm{Ga}$ or In.

\section{INTRODUCTION}

Recently, it was reported by some of the present authors [1] that superconductivity can occur in GaAs crystals. Strong hints for the existence of superconductivity followed from magnetic measurements such as magnetic field modulated microwave absorption (FMMA) and static magnetic susceptibility, which yielded a transition temperature from the normal to the superconductive phase of about 10K [1]. These experiments had been carried out on so-called low-temperature (LT) GaAs, i.e. GaAs layers grown at low temperatures $\left(<200^{\circ} \mathrm{C}\right)$ by molecular beam epitaxy (MBE) [2,3].

Since discovery of a superconductive signal was observed in samples with defective annealed LT-layers it was not clear from which part of the sample signal was generated. Systematic studies were performed to identify a superconductive phase. Layer by layer was removed chemically starting from the top of LT-GaAs layer and a superconductive signal persisted even for samples from which all the LT-layer was removed. Further experiments established that the signal was related to In used to bond the GaAs wafer to a Mo block, and that In must be a fast diffuser in GaAs since more than $100 \mu \mathrm{m}$ of the GaAs wafer was removed prior to the first microwave absorption measurements. This finding was reported in the earlier communication [4]. 
The purpose of this work was to investigate In interaction with GaAs and identify the phase which can be responsible for the FMMA signal.

\section{EXPERIMENTAL DETAILS}

To study the effects related to In diffusion two different types of GaAs samples were prepared. In the first case GaAs bulk material was covered by a metallic In layer. The crystals were then annealed in an $\mathrm{MBE}$ chamber at temperatures between 400 and $550^{\circ} \mathrm{C}$ for $1 \mathrm{~h}$. In the second case, GaAs layers of 10 and $100 \mathrm{~nm}$ thickness were grown by $\mathrm{MBE}$ also at temperatures between 400 and $550^{\circ} \mathrm{C}$ (without In bonding on the back side of the wafe $i$; on a GaAs buffer layer. Deposition of thin In layers (thickness: 1 to $10 \mathrm{~nm}$ ) was attempted by closing the As source and opening an In source. However, growth of InAs was probably the result since normally there exists an As overpressure in the MBE chamber. 4 to 10 periods of these $\mathrm{GaAs} / \mathrm{In}$ (InAs) heterostructure were grown. Subsequently the crystals were thermally annealed at 450 and $550^{\circ} \mathrm{C}$, respectively, for $1 \mathrm{~h}$.

Before electrical measurements were carried out the remaining In on the surfaces of the GaAs bulk crystals was carefully mechanically and chemically removed to avoid further contamination of the crystals by In. Magnetic field modulated microwave absorption was carried out in a Bruker 200 spectrometer over the temperature range from $300 \mathrm{~K}$ to $4 \mathrm{~K}$, and static magnetic susceptibility measurements were made using a SQUID S $600 \mathrm{C}$ magnetometer. The susceptability was measured from $40 \mathrm{~K}$ to $2.5 \mathrm{~K}$.

The morphologies of the samples were characterized by optical microscopy, energy dispersive $x$-ray spectroscopy (EDX) and transmission electron microscopy (TEM). For the second type of samples plan-view as well as cross-section thin foils from different depth regions were prepared by the usual techniques (chemical and ion-beam thinning). The TEM observations were carried out on a JEM 200CX microscope and on the Atomic Resolution Microscope (ARM) at the Lawrence Berkeley Laboratory. The element analysis was done on a JEM 200FX analytical microscope.

\section{RESULTS AND DISCUSSION}

The microwave absorption experiments (FMMA) gave strong hints for the existence of a superconductive phase in the In diffused GaAs bulk material as well as in the GaAs/In(As) heterostructures. A strong FMMA signal was observed near zero field [1,4,5]. In addition to this signal, a local maximum in FMMA amplitude was observed close to 300 Gs. This was in agreement with earlier observations [1,4]. The phase transition took place close to $7 \mathrm{~K}[5]$, slightly lower than that determined earlier (more precise temperature measurements). Above $7 \mathrm{~K}$ the signal disappeared completely.

For the GaAs bulk material optical microscopy showed, after removing of the In layer, that the surfaces contained two types of defects as shown in Fig. 1. Large inclusions were present with diameters of about $50 \mu \mathrm{m}$ and a density in the order of $10^{5} \mathrm{~cm} .^{-2}$ Element analysis using EDX indicated that these large particles consisted of pure In, or an In-rich compound. Such inclusions were found in the samples down to a depth of about $100 \mu \mathrm{m}$. The other type of surface defect was a small scale roughness or etch pits. Their density also decreased with increasing depth from the In covered surface, however, their density was at least two orders of 


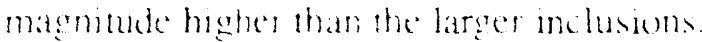

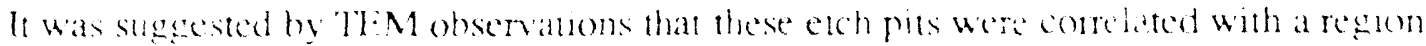
of high dislexation demsity. These dislexations shat to form from the In corered bulh surface

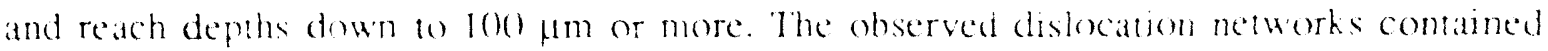
bundles of clustered distexatons and long individual dislexation lines as shown in figs. 2 and ? respectively:

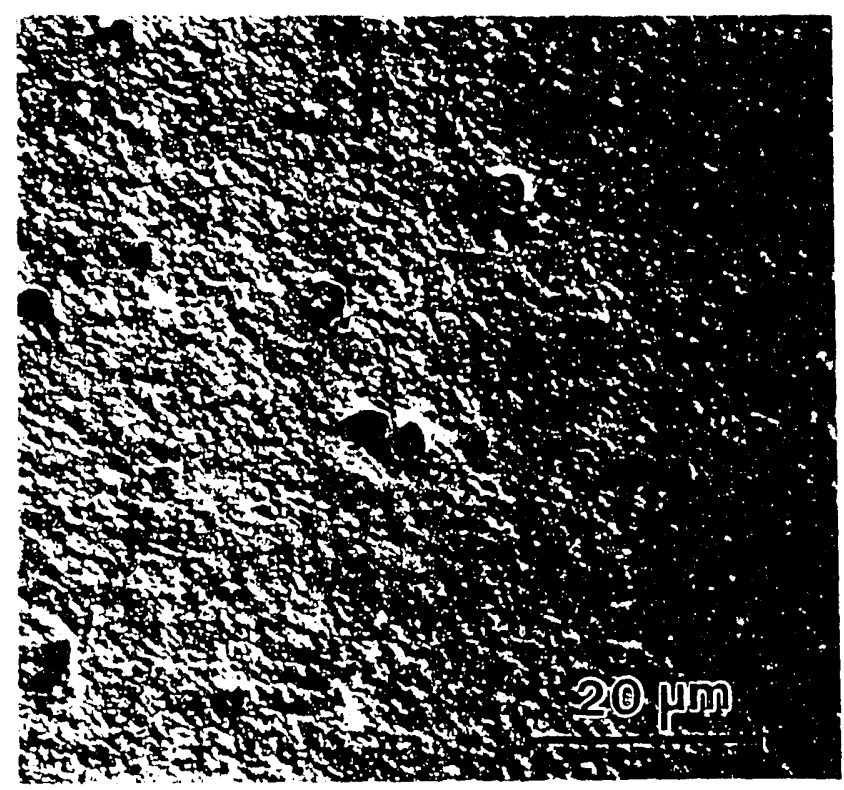

Fig. 1. Light-optical micrograph of the chemically etched back side of a I.T-CiaAs wafer at the depth of $40 \mathrm{~km}$ below the surface. Two different lypes of defects are visible: large metallic inclusions ( $\varnothing$ * $10 \mu \mathrm{m})$ and small etch pits $(\varnothing$ $<100 \mathrm{~mm}$ ) having an average distance of $1 \mu \mathrm{m}$.
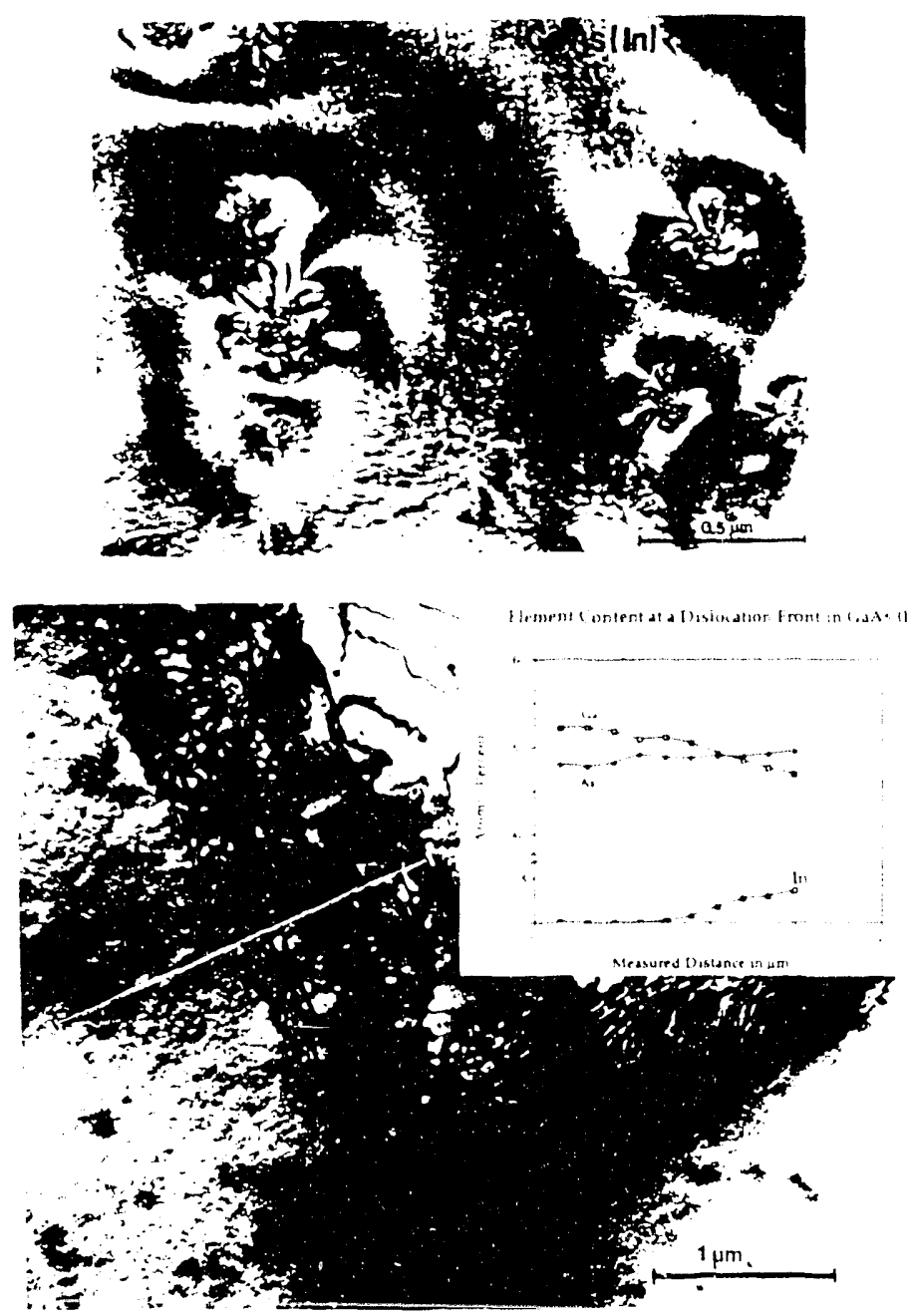

Fig. 2. TEM nicrograph of a corresponding region shown in Fig. 1. Characteristic features areas with a diameter of $(\varnothing<0.5$ $\mu \mathrm{m})$ are highly strained. The core of the defects seems to consists of a cluster of dislocations.

Fig. 3. TEM micrograph of a dislocation front in an In diffused GaAs wafer The insert shows the result of an EDX analysis related to changes of the In content across the transition from the defect free bulk to the dislocation network. 
EDX analysis showed the existence of indium segregation in these dislocation clusters and indiv sual lines. The insert in Fig. 3 shows In concentration obtained from a line scan across the dislocation front. The As content remains nearly constant, whereas the Ga content slightly decreases. Indium precipitates were not observed in this area.

In the following the results of the TEM investigation of the heterolayer structures are presented. Fig. 4 shows a cross-section image of the multi-layer system, which consisted of 4 layers of GaAs (thickness about $100 \mathrm{~nm}$ ) and dark layers of about $10 \mathrm{~nm}$ thickness between them corresponding to those films which should consist of In. However, In layers were not found in these samples. The dark horizontal bands contained strongly straineci regions. The inclined lines which originated from these black bands correspond to stacking faults and microtwins indicating lattice-mismatch.

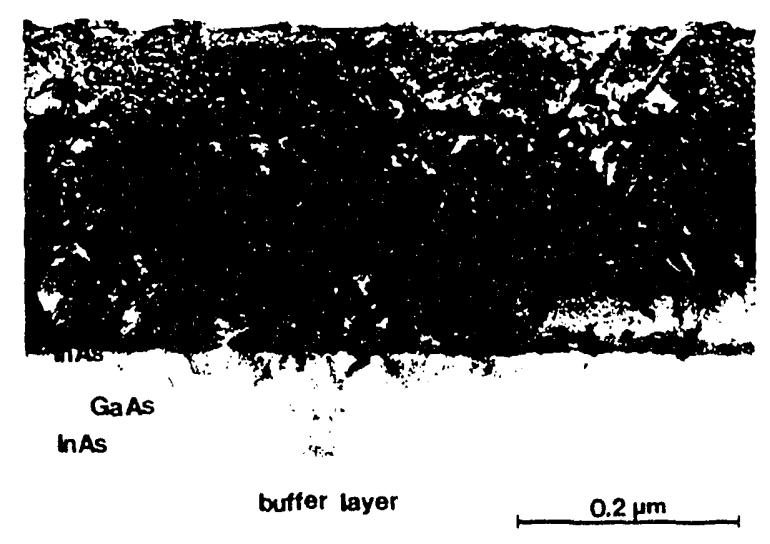

Fig. 4. Cross-section TEM image of an $\operatorname{InAs}(10 \mathrm{~nm}) / \mathrm{GaAs}(100 \mathrm{~nm})$ multi-layer grown by MBE. Three-dimensional growth of InAs islands can clearly be observed at the surface. Micro twins (inclined dark lines) are generated to reduce the lattice misfit. Viewing direction: $<110>$.

Corresponding HREM lattice images revealed that there existed lenticular inclusions of InAs between the GaAs layers (Fig.5a). Periodic strain fields as seen in the interface with an InAs particle were identified as Lomer-dislocations. As typical growth features the lentilcular InAs particles were often connected by pipe-like thin layers surrounded by strain fields. Often the particles occurred isolated as islands as demonstrated in Fig. $5 \mathrm{~b}$.
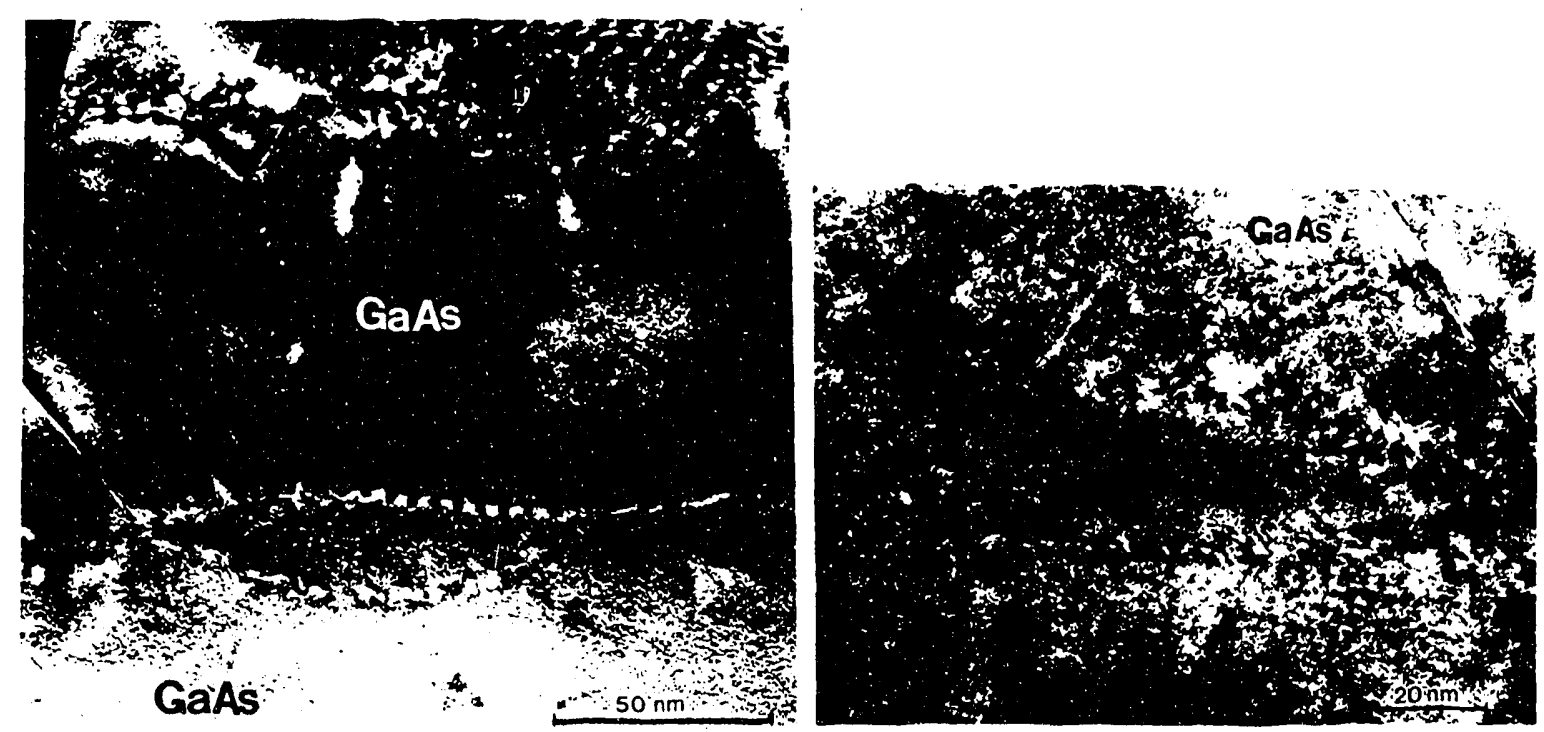

Fig. 5. (a) Magnified cross-section image showing the morphology of the InAs inclusions; (b) Isolated, lens -shape InAs particle in the GaAs matrix as the result of a 3-dimensional MBE growth at $500^{\circ} \mathrm{C}$. 
In conclusions, it was shown that In is a very fast diffuser and can penetrate a GaAs sample for more than $100 \mu \mathrm{m}$ during $1 \mathrm{~h}$ annealing in the temperature range $4(1)$ to $550^{\circ} \mathrm{C}$. This diffusion is associated with the formation of the high density of dislocations and stacking faults. Since In is often used to bond wafers in MBE growth, it is expected that some properties of these wafers might be affected. Indium deposition in the form of In/GaAs superlayers leads to the formation either InGaAs ternary compounds or InAs layers, both highly stressed.

Both types of samples (with In deposited on the GaAs surface and In-interlayers, subsequently annealed) showed microwave absorption signals typical for superconductivity. However, it is difficult to relate the FMMA signal with the observed morphologies because no likely superconductive phase was found in either kind of specimen. However, samples which contained isolated strained inclusions of InAs showed much stronger FMMA signals than the samples where InAs was a continuous layer. Independent studies confirmed existence of superconductivity in In diffused GaAs [6]. Therefore, the most likely origin of the FMMA signal in our samples are strongly distorted or amorphous inclusions related to the excess group-III elements. It is worth to note that amorphous In and $\mathrm{Ga}$ have superconductive transition temperatures of $5 \mathrm{~K}$ and $7 \mathrm{~K}$, respectively [7]. However, these amorphous phases have recrystallization temperatures below $200 \mathrm{~K}$. Thus it might be the stabilization of such highly distorted inclusions within the GaAs matrix which is the key to the observed superconductivity effects.

\section{ACKNOWLEDGMENT}

This work was supported by the Director, Office of Energy Research, Office of Basic Energy Sciences, Materials Sciences Division, of the U.S. Department of Energy under Contract No. DE-AC03-76SF00098. The use of the facility of the National Center for Electron Microscopy in the Lawrence Berkeley Laboratory is greatly appreciated. P.W. also wants to thank the Alexander von Humboldt Foundation/ Germany for providing him with financial al support during his stay as a visiting scientist at LBL Berkeley.

\section{REFERENCES}

1. J.M. Baranowski, Z.Liliental-Weber, W.-F. Yau, E.R. Weber, Phys. Rev. Lett., 66, 3079 (1991)

2. Z.Liliental-Weber, W. Swider, K.M. Yu, J.B. Kortright, F.W. Smith, and A.R. Calawa, Appl. Phys. Lett., 58, 2153 (1991).

3. Z.Liliental-Weber, A. Claverie, P. Werner, W. Schaff, and E.R. Weber, in "Materials Science Forum, Defects in Semiconductors," Vol. 83-87, 1045 (1992).

4. J.M. Baranowski, Z.Liliental-Weber, W.-F. Yau, E.R. Web:r, Phys.Rev.Lett., 68, 551 (1992).

5. J.M. Baranowski, P. Wojcik, M. Palczewska, R. Jablonski, E.R. Weber, W.-F. Yau, H. Sohn, and P. Werner, Phys. Rev. Lett., Acta Physica Polonica A, vol. 82, 670 (1992).

6. Y.K. Li, Y. Huang, Z. Fan, C. Jiang, X.B. Mei, B. Yin, J.M. Zhou, J.C. Mao, J.S. Fu, and E. Wu, J. Appl. Phys., 71, 2018 (1992).

7. Landolt-Bernstein, Numerical data and Functional Relationship in Science and Technology, New Series, vol. III 21 a, Spring - Verlag, Berlin (1989). 

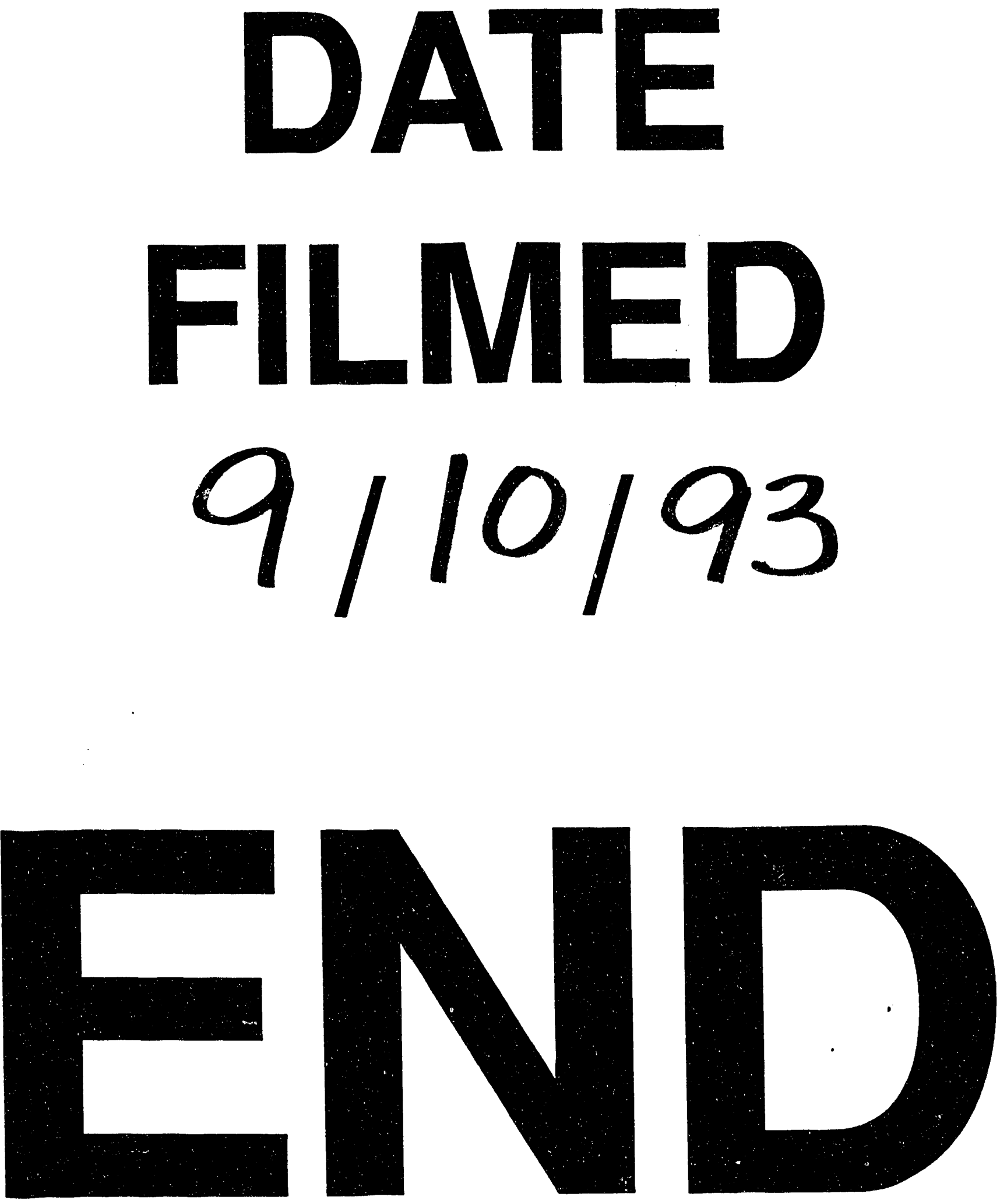
